\title{
ФЛУКТУАЦИИ ПРИМЕСНОГО ПОГЛОЩЕНИЯ И ИЗЛУЧЕНИЯ ПРИ НИЗКОЙ ТЕМПЕРАТУРЕ
}

\author{
V. HIZNJAKOV. LISANDINEELDUMISE JA -KIIRGUSE FLUKTUATSIOONID MADALAL TEM- \\ PERATUURIL \\ \%. HIZHNYAKOV. FLUCTUATIONS OF IMPURITY ABSORPTION AND EMISSION AT LOW \\ TEMPERATURE
}

Спектроскопические исследования отдельных атомов и молекул в свободном (газообразном) состоянии ведутся уже ряд лет (см., напр., $\left.\left[{ }^{1,2}\right]\right)$. В этих ислледованиях используются атомы и молекулы, разделенные пространственно на значительное расстояние (что и позволяет их наблюдать по отдельности). В твердом теле примесные центры находятся на очень малых расстояниях друг от друга, гораздо меньших дифракционного предела фокусировки света $\lambda(\lambda-$ длина волны света). Поэтому может показаться, что оптический эксперимент на отдельном центре (или на небольшом их числе) 'в кристалле не возможен. В действительности это не так: существование узких бесфононных линий шириной, гораздо меньшей наблюдаемой (неоднородной) ширины, делает такие эксперименты при низкой температуре в принципе возможными. На это ранее указывал К. К. Ребане в связи с обсуждением вопроса о предельно достижимой плотности записи информации в твердых телах. Ниже предлагается один из возможных путей реализации этой идеи.

Рассмотрим кристалл (стекло), оптическая толщина которого $(\boldsymbol{x})$ в области бесфононной линии (БФЛ) примесей порядка единицы $(x \sim 1)$. Понизим температуру кристалла настолько, чтобы однородная ширина БФЛ $(\gamma)$ определялась скоростью $\left(\gamma_{0}\right)$ распада возбужденного состояния центров $\left(\gamma \approx \gamma_{0}\right)$ (в кристаллах для этого обычно достаточно использовать температуру $\left.\sim 1 \mathrm{~K}\left[{ }^{3}\right]\right)$. Зададим вопрос, сколько примесных центров будет участвовать в поглощении монохроматического луча сечения $S \sim d^{2}(d-$ диаметр луча; спектральная ширина излучения $\left.\delta<\gamma_{0}\right)$ ? Простая оценка, основанная на формуле

$$
x \sim \bar{\gamma}_{0} p l \lambda^{2} / 2 \pi \Gamma
$$

$\left(\bar{\gamma}_{0}=\gamma_{0} \exp (-f), \exp (-f) \quad\right.$ - относительная вероятность БФЛ, $p-$ концентрация центров, $l$ - толщина кристалла, $\Gamma-$ неоднородная ширина БФЛ, $\lambda^{2} / 2 \pi$ - сечение резонансного поглощения одного центра) дает для этого числа $(n)$ величину

$$
n=p l S \bar{\gamma}_{0} / \Gamma x \sim 2 \pi d^{2} / \lambda^{2} .
$$

Возбуждающее излучение можно сфокусировать на площадь $d^{2} \sim \lambda^{2}$ (дифракционный предел). В этом случае $n \sim 2 \pi$, т. е. возбуждается и тем самым участвуег в поглощении только несколько центров.

При таком малом числе поглощающих центров, очевидно, весьма существенны эффекты флуктуации: при изменении частоты возбужде- 
ния всего лишь на величину $\sim \gamma_{0}$ (либо при перефокусировке на расстояние $\sim d$ ) число поглощающих центров будет нерегулярным (случайным) образом изменяться на величину $\sim n^{1 / 2}$. Это приведет к изменению коэффициента поглощения на относительную величину $\Delta x / x \sim n^{-1 / 2}$. Поэтому коэффициент поглощения в пределах неоднородной БФЛ будет флуктуировать с относительной амплитудой $\sim n^{-1 / 2}$ при спектральной «длине» корреляции $\sim y_{0}$. Если фокусировка излучения на кристалл не полная, а ширина БФЛ отличается от радиационной, то относительная амплитуда флуктуаций коэффициента поглощения уменьшается до величины

$$
\frac{\Delta x}{x} \sim \frac{\lambda \overline{\gamma_{0}^{1 / 2}}}{d(2 \pi(\gamma+\delta))^{1 / 2}}
$$

при спектральной «длине» корреляции $\sim \gamma+\delta$.

Следует отметить, что флуктуации коэффициента поглощения отмеченной величины будут наблюдаться только в том случае, когда центр после поглощения одного фотона возбуждения успевает высветиться и вернуться в основное состояние еще до прихода следующего фотона. Это дает следующее ограничение на интенгивность возбуждения сверху:

$$
I<\left(2 \pi \gamma_{0}(\gamma+\delta)\right)^{1 / 2} d / \lambda
$$

В системах с фотовыжиганием спектрального провала помимо отмеченного ограничения на интенсивность возникают также ограничения на дозу $N$ (полное число фотонов) и квантовый выход фотовыжигания $\alpha$ :

$$
\begin{gathered}
N=I t<2 \pi d^{2}(\gamma+\delta)^{1 / 2} / \overline{\gamma_{0}^{1 / 2}} \alpha \lambda^{2}, \\
\alpha \ll 1
\end{gathered}
$$

( $t$ - время).

Проведем оценку. Если $\gamma+\delta \sim \gamma_{0} \sim 5 \cdot 10^{7} \quad$ сек $^{-1}, d \sim 10^{-3}$ см, $\lambda \sim 5 \cdot 10^{-5}$ см, $\alpha \sim 10^{-5}$, то $I<10^{9}$ сек $^{-9}, N<10^{8}$. В этом случае относительная амплитуда полезного сигнала (флуктуаций коэффициента поглощения) порядка нескольких процентов $\left(\Delta x / x<10^{-2}\right)$, т. е. существенно превышает уровень квантового шума регистрации $\sim N^{-1 / 2} \sim 10^{-4}$.

Рассмотренный выше достаточно очевидный эффект флуктуаций чиєла поглощающих центров, возникающих при фокусировке слабого монохроматического излучения, может быть использован для изучения различных релаксационных процессов в твердых телах при низкой температуре. В частности, таким методом можно изучать процессы спектральной диффузии в стеклах. При этом может быть зафиксирован отдельный квантовый переход в ближайшей к одному из поглощающих центров двухуровневой системе: такой переход приводит к выводу центра из резонанса, т. е. к скачкообразному изменению числа поглощающих центров на единицу. Это уменьшает коэффициент поглощения на относительную величину $\sim \lambda^{2} \overline{\gamma_{0}} / 2 \pi(\gamma+\delta) d^{2}$. Отметим также, что предлагаемым методом можно наблюдать индивидуальные процессы фотовыжигания провала (если $\alpha \ll 1)$ : каждый акт фотопревращения также приводит к скачку (уменьшению) поглощения на относительную величину $\sim \lambda^{2} \overline{\gamma_{0}} / 2 \pi(\gamma+\delta) d^{2}$, который может быть зафиксирован.

Рассмотренные выше флуктуационные эффекты могут наблюдаться не только в поглощении, но и в резонансной флуоресценции, возбуждаемой сфокусированным монохроматическим светом ограниченной интенсивности. При этом величина флуктуаций может быть в $x^{-1 / 2}$ раз уси- 
лена по сравнению с поглощением, если использовать кристалл с малой оптической толщиной $x$. Например, если $x \sim 10^{-2}, d \sim 5 \mu$, то возбуждаются и излучают одиночные центры и амплитуда флуктуаций регистрируемого излучения $\Delta I \sim I$.

Еще одна возможность усиления флуктуаций примесных центров состоит в использовании двойной фокусировки - как возбуждения, так и регистрации. В этом случае число центров, излучение которых регистрируется, равно

$$
n \sim p(\gamma+\delta) d^{2} d_{1}^{2} / \Gamma\left(d+d_{1}\right)
$$

$\left(d_{1}-\right.$ диаметр фокусировки при регистрации). Принимая $(\gamma+\delta) / \Gamma \sim$ $\sim 10^{-6}, p \sim 10^{16} \mathrm{~cm}^{-3}, d \sim d_{1} \sim 5 \mu$, получаем $n \sim 1$, т. е. будет регистрироваться излучение одиночных центров. При этом снова относительная амплитуда флуктуаций будет порядка единицы. Отметим также, что в рассматриваемых экспериментах по резонансной флуоресценции примесей могут наблюдаться корпускулярные флуктуации не только в веществе, но и в поле излучения, например, антигруппировка фотонов $\left[{ }^{4}\right]$.

В заключение отметим, что предлагаемый флуктуационный метод реализует спектроскопию небольшого числа примесных центров кристаллов при низкой температуре.

Автор признателен К. К. Ребане за обсуждение.

\section{ЛИ Т Е Р А Т У Р А}

1. Демтредер В. Лазерная спектроскопия. М., Наука, 1985, 537-561.

2. Летохов В. С. Применение лазеров в атомной, молекулярной и ядерной физике. Тр. Всесоюзн. школы. М., Наука, 1979.

3. Хижняков В. В. // Тр. ИФ АН ЭССР, 1986, № 59, 55-74, Тарту.

4. Смирнов Д. Е., Трочин А. С. // УФН, 1987, 153, 233-271.
Институт физики
Академии наук Эстонской ССР
Поступила в редакцию
$13 / \mathrm{V} \quad 1988$ 